\title{
Long valley relaxation time of free carriers in monolayer $\mathrm{WSe}_{2}$
}

\author{
Tengfei Yan, Siyuan Yang, Dian Li, and Xiaodong Cui* \\ Department of Physics, The University of Hong Kong, Pokfulam, Hong Kong \\ (Received 23 November 2016; revised manuscript received 22 May 2017; published 20 June 2017)
}

\begin{abstract}
Monolayer transition metal dichalcogenides (TMDs) feature a valley degree of freedom, giant spin-orbit coupling, and spin-valley locking. These exotic natures have stimulated efforts of exploring potential applications in conceptual spintronics, valleytronics, and quantum computing. Among all the exotic directions, a long relaxation time of spin and/or valley polarization is critical. The present valley dynamics studies concentrate on the band edge excitons which predominate the optical response due to an enhanced Coulomb interaction in two dimensions. The valley relaxation time of free carriers remains ambiguous. In this Rapid Communication, we use time-resolved Kerr rotation spectroscopy to probe the valley dynamics of excitons and free carriers in monolayer tungsten diselenide. The valley relaxation time of free carriers is found around $2 \mathrm{~ns}$ at $70 \mathrm{~K}$, about three orders of magnitude longer than the excitons of about $2 \mathrm{ps}$, and 15 times larger than that of trions (130 ps). The extended valley relaxation time of free carriers evidences that an exchange interaction dominates the valley relaxation in optical excitations. The pump-probe spectroscopy also reveals an exciton binding energy of $0.60 \mathrm{eV}$ in monolayer $\mathrm{WSe}_{2}$.
\end{abstract}

DOI: 10.1103/PhysRevB.95.241406

In solid state physics, a valley refers to the local energy extreme, either a conduction band local minimum or valence band local maximum, in crystal electronic band structures. The occupation of carriers at inequivalent valleys, carrying different momentum phases, represents different quantum states. This leads to conceptual valleytronics, which utilizes the valley degree of freedom as a quantum information carrier, in a similar way as the spintronics where a spin degree of freedom is utilized [1-4]. Monolayer transition metal dichalcogenides (TMDs), an emerging two-dimensional (2D) semiconductor, feature degenerate but inequivalent valleys $K$ and $K^{\prime}$ (or $-K$ ) located at the band edges of both conducting and valence bands, which are separated by a large momentum space. Owing to spatial inversion symmetry breaking in monolayer TMDs, the Berry curvature, a function describing the properties of valence electron orbits in crystal lattices, shows opposite signs at the $K$ and $K^{\prime}$ valleys. This could work as a knob to selectively manipulate the $K$ or $K^{\prime}$ valley [5-9]. Besides, $K$ and $K^{\prime}$ valleys are constructed by the metal's $d$ orbits, which experience strong spin-orbit coupling (SOC). The SOC splits the band, particularly the valence band, into two subbands. As a result of time-reversal symmetry, the spin splitting shows opposite signs between the $K$ and $K^{\prime}$ valleys at equal energies [5,10-12]. Namely, if the band edge at the $K$ valley is a spin-up state, the band edge at the $K^{\prime}$ valley must be a spin-down state, as illustrated in Fig. 1(a). This leads to a definite relationship between the valley and spin indices, the so-called spin-valley locking [13-15]. This unique spin-valley locking interplays the valley and spin degrees of freedom and suppresses the valley/spin relaxation: In the relaxation process of a hot carrier around a $K$ valley, the conservation of momentum (valley) and spin both must be satisfied. It theoretically supports a long valley/spin relaxation time in monolayer TMDs.

On the experimental side, however, the valley/spin relaxation time of free carriers remains ambiguous. Spin-

\footnotetext{
*Author to whom correspondence should be addressed: xdcui@hku.hk
}

resolved photocurrent measurements estimated the valley/spin relaxation time in the range of $10^{0}-10^{2} \mathrm{~ns}$ in monolayer $\mathrm{WS}_{2}$ [16], while optical pump-probe spectroscopy and timeresolved photoluminescence (PL) experiments gave a very short valley relaxation time of several picoseconds [17-25]. Long valley relaxation times of bound excitons or residue holes were reported [26-30]. The huge discrepancy lies in the fact that the excitonic effect is prevalent in the optical responses of monolayer TMDs [31-37]. The giant exciton binding energy implies a short effective radius of the excitons, a close separation between electrons and holes, enhancing the exchange interactions. Spin exchange interactions are believed to be the major valley/spin relaxation channel in monolayer TMDs that causes the short valley/spin relaxation time of excitons [23,38-40]. To date, the direct measurement of free carriers is lacking. In this Rapid Communication, we use time-resolved Kerr rotation spectroscopy to unambiguously identify the valley/spin relaxation time of free carriers in monolayer $\mathrm{WSe}_{2}$. It is three orders of magnitude longer than that of excitons. The results show that monolayer TMD is a promising platform for conceptual valleytronics and nonmagnetic spintronics.

$\mathrm{WSe}_{2}$ flakes are mechanically exfoliated from singlecrystal $\mathrm{WSe}_{2}$ onto $\mathrm{Si} / \mathrm{SiO}_{2}$ substrates for PL and pump-probe measurements, and mica for transmittance measurements. The two-color pump-probe measurement setup is shown in Fig. 1(b). More details of the helicity-resolved PL and the pump-probe measurement setups have been illustrated elsewhere $[6,41,42]$. Monolayer $\mathrm{WSe}_{2}$ flakes are identified by an optical microscope and PL.

The optical interband transitions are characterized by PL and absorption spectroscopy at room temperature, as shown in Fig. 1(c). There are three prominent absorption peaks, consistent with previous reports [43], which are labeled as $\mathrm{A}, \mathrm{B}$, and C. Peaks A and B correspond to the two exciton states of spin-split interband transitions at the $K$ and $K^{\prime}$ valleys. Peak $\mathrm{C}$ corresponds to several exciton states of the interband transition near the $\Lambda$ and $\Gamma$ points in the Brillouin zone as a result of the band nesting effect $[44,45]$. The energy 
(a)

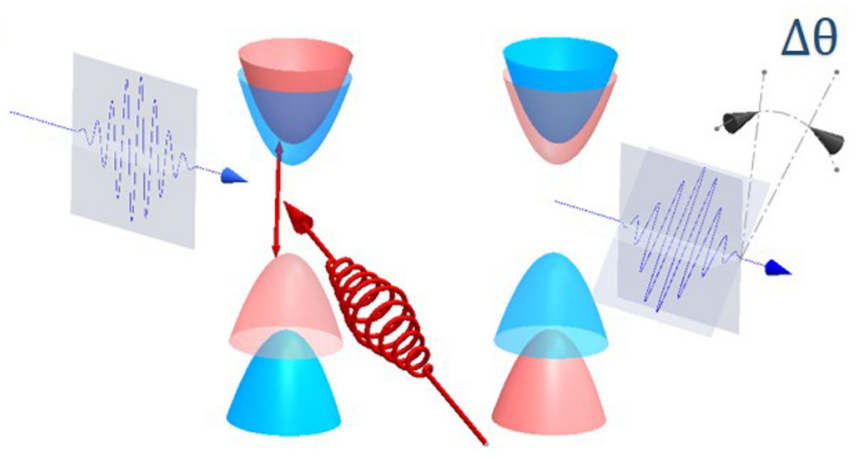

(b)

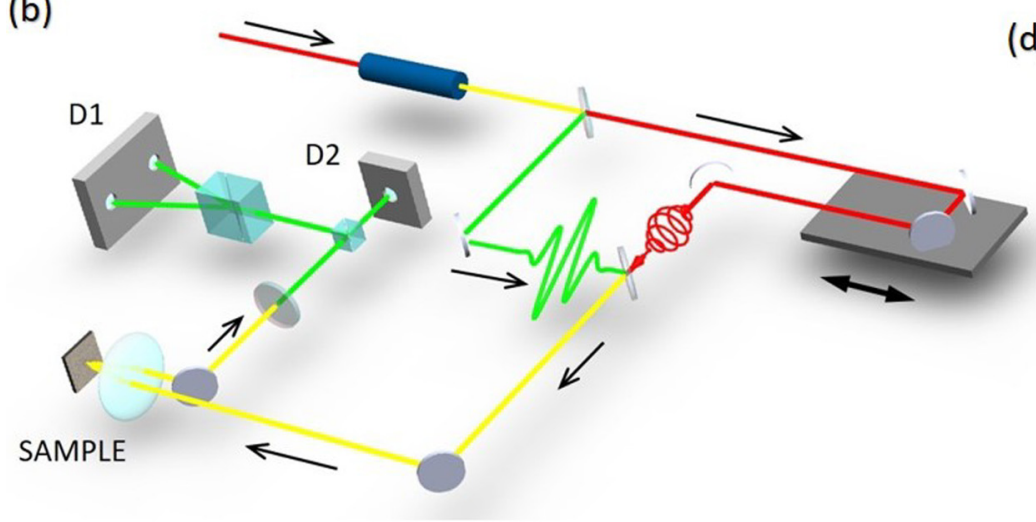

(c)

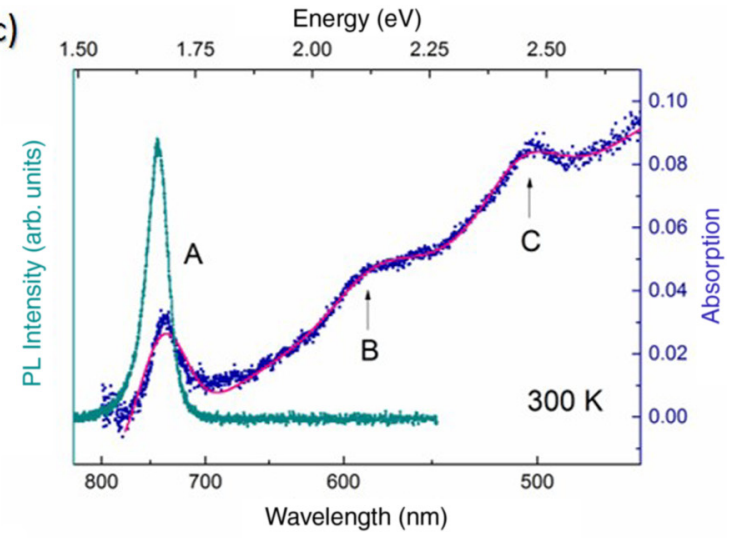

(d)

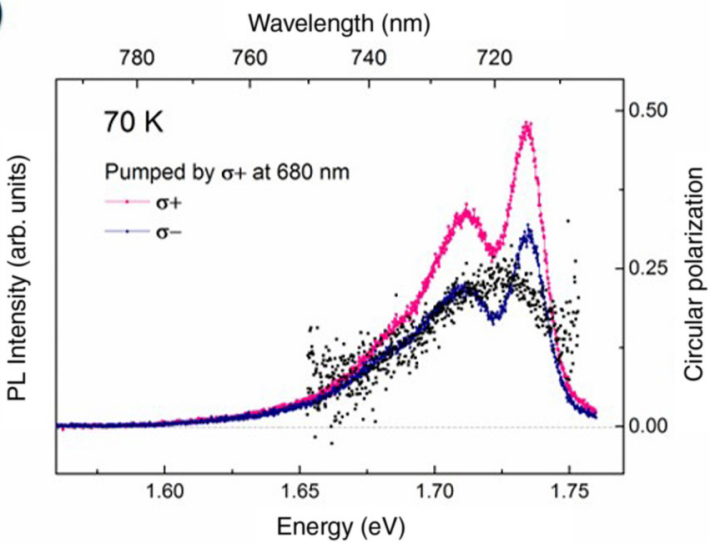

FIG. 1. (a) A schematic diagram of the monolayer $\mathrm{WSe}_{2}$ band structure in the $K$ (left) and $K^{\prime}$ (right) valleys, their spin states (shown in red and blue colors, respectively), and the light-matter interaction in a time-resolved Kerr rotation experiment. (b) Schematic of pump-probe Kerr rotation spectroscopy. D1 is a balanced photodetector and D2 a photodiode, working together to get the Kerr rotation as an optical bridge. D2 is a photodiode monitoring the reflectivity change. (c) PL spectrum of the monolayer $\mathrm{WSe}_{2}$ on a Si/SiO${ }_{2}$ substrate (green dots), and absorption spectrum on mica (the blue dots) with a Gaussian function fit (red line). The data are measured at room temperature. (d) Helicity-resolved PL spectra of monolayer $\mathrm{WSe}_{2}$ pumped by a $1.824 \mathrm{eV}$ left-handed circularly polarized laser $(\sigma+)$ at $70 \mathrm{~K}$. The PL circular polarization, calculated by $\left(I_{\sigma+}-I_{\sigma-}\right) /\left(I_{\sigma+}+I_{\sigma-}\right)$, is also shown in the figure by the black dots.

shift of the A exciton between the absorption and PL spectra shows a Stokes shift, indicating the unintentional doping in the material [8]. Different exciton binding energies modified by the dielectric permittivity of the mica and $\mathrm{SiO}_{2}$ substrates may also contribute to the energy shift $[36,46]$.

Figure 1(d) shows the helicity-resolved PL spectra of a $\mathrm{WSe}_{2}$ flake excited by a left-handed circularly polarized laser $(\sigma+$, right-handed circularly polarized is represented as $\sigma-)$ at $70 \mathrm{~K}$. The emission peak at $1.734 \mathrm{eV}$ and the lower energy peak at $1.712 \mathrm{eV}$ are identified as the A excitons and the charged excitons (trions). The collected PL signal shows a prominent circular polarization of about $24 \%$ for both excitons and trions, indicating a clear valley polarization in the $K$ and $K^{\prime}$ valleys. The lower energy tail, consisting of defect bound excitons, shows negligible valley polarization.

We measured the valley relaxation of $\mathrm{A}$ excitons by pumping the sample at $1.834 \mathrm{eV}$ and probing at $1.737 \mathrm{eV}$ (pumping A, probing A). The probing beam intensity is tuned to be a tenth of the pumping beam to minimize its influence. The pumping beam injected exciton density is estimated to be at the magnitude of $10^{12} \mathrm{~cm}^{-2}$, given the absorption ratio shown in Fig. 1(c) and the laser spot radius of $1 \mu \mathrm{m}$. Both data with left-handed and right-handed circularly polarized pumping beams are shown in Fig. 2(a). The valley polarization relaxations are well described with single exponential decay functions. The valley relaxation time is extracted to be $1.8 \pm 0.3 \mathrm{ps}$, consistent with previous studies [23,42]. Such a quick relaxation process is attributed to exciton intervalley $\left(K-K^{\prime}\right)$ scattering through the strong electron-hole exchange interaction, which is diagramed in the inset of Fig. 2(a) $[38,39]$. The pump energy is tuned slightly larger than the A exciton $1 s$ transition energy due to the experiment's limitation. The valley relaxation time dependence on pump energy in $\mathrm{WSe}_{2}$ is rather weak, as shown in Fig. S3 [47]. This may be related to the dark exciton, which characterizes a smaller energy than the bright exciton, resulting in pumped excitons that easily relax to the dark exciton state [48-50].

The time-resolved reflection spectrum with the same pumping and probing energy is shown in Fig. 2(d). The biexponential decay function fit shows the $\Delta R$ decay time constants are $5 \pm 0.1$ and $25 \pm 3$ ps. The fast decay may be related to the phase space redistribution via exciton-exciton annihilation [51]. More details may be found in the Supplemental Material [47]. The exciton formation time is deduced to be less than $0.5 \mathrm{ps}$, limited by the present time resolution of the experimental setup. 

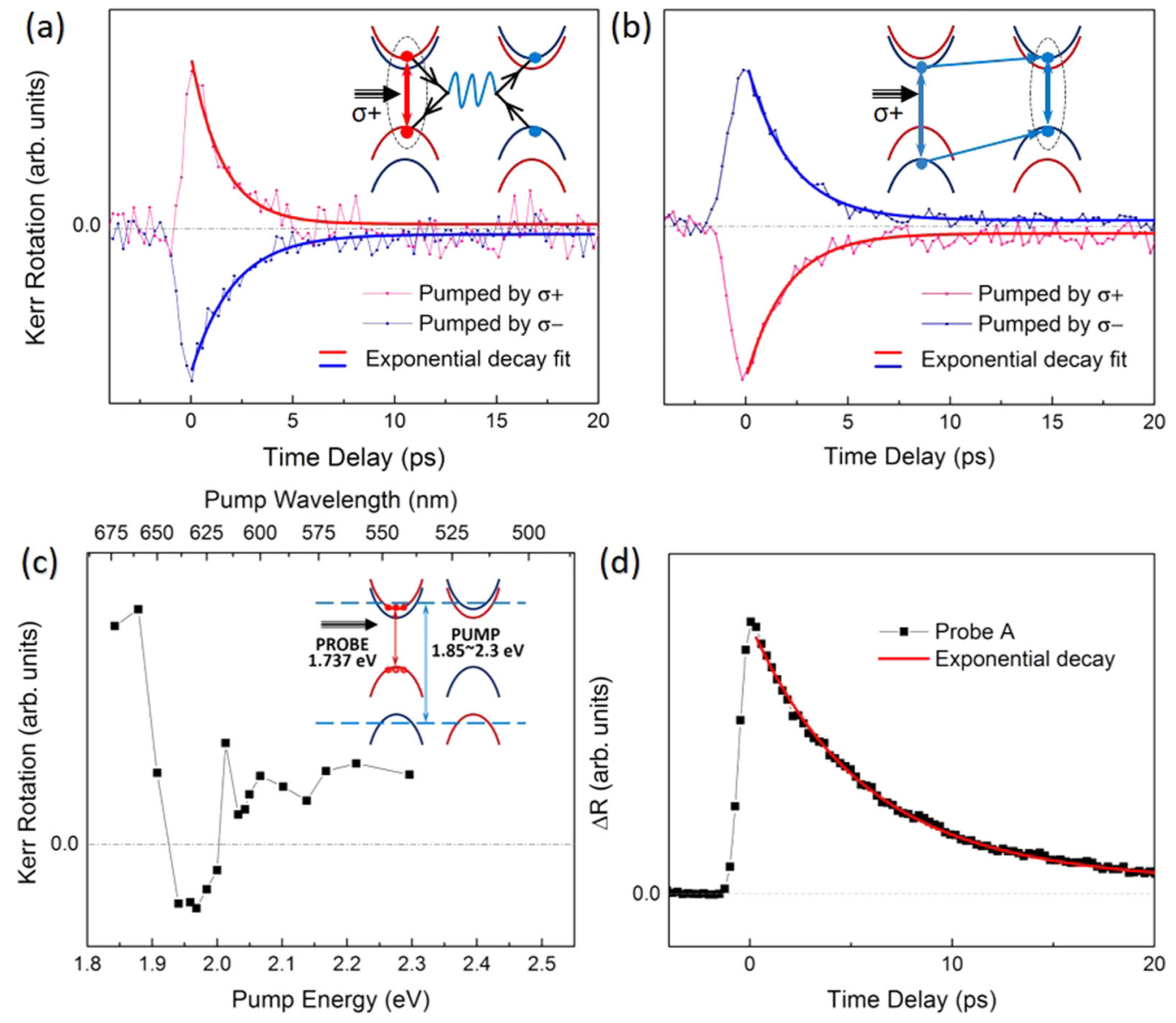

FIG. 2. (a), (b) Time-resolved Kerr rotation of monolayer $\mathrm{WSe}_{2}$ measured at $70 \mathrm{~K}$. The red and blue dotted lines indicate the Kerr rotation traces when pumped by left-handed (red) and right-handed (blue) circularly polarized pulses, respectively. The solid lines follow an exponential decay fit. (a) The sample is pumped by the pulse centered at $1.834 \mathrm{eV}$ and probed at $1.737 \mathrm{eV}$ (pumping A, probing A). Please refer to the Supplemental Material [47] for the pump-energy-dependent valley relaxation time. The insets illustrate the band structure and transitions between different bands. (b) The sample is pumped at $2.023 \mathrm{eV}$ and probed at $1.737 \mathrm{eV}$ (pumping B, probing A). The sign change in the Kerr rotation signal implies that the B excitons first relax to A excitons via intervalley scatterings, shown in the inset. (c) The Kerr rotation angle at zero time delay as a function of the pumping energy. (d) The time-resolved reflectance spectrum probed at $1.737 \mathrm{eV}$ and pumped at $1.834 \mathrm{eV}$, with an exponential decay function fit in the red solid line.

While we tune the pumping energy to $2.023 \mathrm{eV}$, near resonant with $\mathrm{B}$ excitons, the Kerr rotation at $1.737 \mathrm{eV}$ (pumping B, probing A) changes its sign, as shown in Fig. 2(b). This implies that spin-conserved intervalley scattering prevails over spin-flip intravalley scattering in the hot carrier relaxation process, as illustrated in the inset of Fig. 2(b). For B excitons generated by a left-handed circularly polarized pump in the $K$ valley, the holes could be scattered to the valence band edge and electrons to the higher spin-split subband of the conduction band at the $K^{\prime}$ valley via Coulomb interactions without a spin flip, forming A excitons at the $K^{\prime}$ valley. Thus a sign change in Kerr rotation is observed. The rise time of the Kerr rotation signal here is approximately 0.8 ps longer than that in the case of pumping A, probing A, possibly resulting from the time of hot exciton intravalley relaxation, intervalley scattering, and the formation of A excitons. The relaxation time is deduced to be $2 \pm 0.2 \mathrm{ps}$, similar to that of the pumping A, probing A situation within the error bar. It is consistent with the proposed relaxation mechanism, for the depolarization process of the A excitons in both cases shares the same relaxation channel, the electron-hole exchange interactions.

The Kerr rotation angle measured at zero time delay is plotted as a function of the pumping energy, which is shown in Fig. 2(c). There is a clear region centered at $1.97 \mathrm{eV}$ that shows that the Kerr rotation exhibits a negative signal. We assign it to the B exciton states while Figs. 1(c) and 1(d) imply an energy of B excitons of around $2.184 \mathrm{eV}$. The large energy shift of about $0.21 \mathrm{eV}$ between the time-resolved and steadystate spectra is attributed to the band-gap renormalization, i.e., the energy lowering correlation of free carriers, caused by the dense exciton density up to $10^{12} \mathrm{~cm}^{-2}$ injected by the pumping pulses in the transient measurement. The energy shift is much larger compared to the quasi-2D systems such as GaAs quantum wells as a result of enhanced electron-electron interactions $[17,52,53]$.

To further study the valley dynamics in monolayer $\mathrm{WSe}_{2}$, we set the pumping energy to be $1.737 \mathrm{eV}$, which is resonant with the A excitons, and tune the probing energy in the range between 1.968 and $2.505 \mathrm{eV}$. The transient differential 
(a) Probe Wavelength $(\mathrm{nm})$

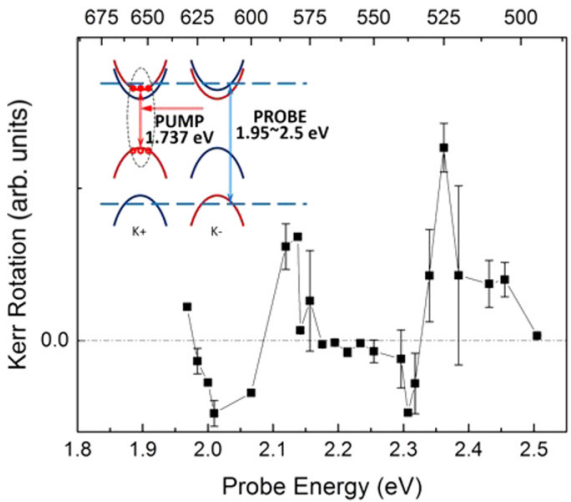

(b)

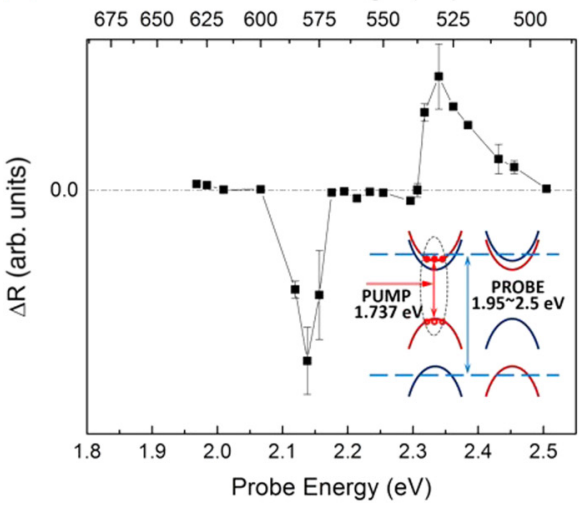

(c)

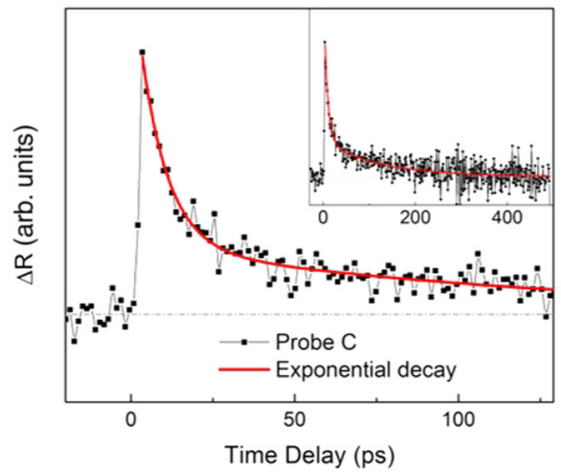

FIG. 3. (a), (b) Transient differential reflection intensity and the Kerr rotation angle at zero time delay as a function of the probing energy. Both experiments are conducted with the pumping laser energy set at $1.737 \mathrm{eV}$ and the probing laser energy tuned from 1.95 to $2.5 \mathrm{eV}$. (c) Time-resolved reflectance pumped at $1.737 \mathrm{eV}$ and probed at $2.318 \mathrm{eV}$. The inset shows the same trace in a larger time scale.

reflections at zero time delay are plotted as a function of the probing energy, as shown in Fig. 3(b). To be noted, signals measured here indicate that higher energy states are occupied, which may be related to Auger-like scatterings. An efficient upconversion from the A exciton to the B exciton has been reported [54]. Negative differential reflection peaking at $2.15 \mathrm{eV}$ is observed, close to $2.184 \mathrm{eV}$ of the $\mathrm{B}$ excitons extracted from steady-state optical measurements. The transition is confirmed at the same energy in Fig. 3(a), in which the probing-energy-dependent Kerr rotation spectrum at zero time delay is shown. The redshift of $0.034 \mathrm{eV}$ of $\mathrm{B}$ excitons is remarkably different from that of $0.21 \mathrm{eV}$ in Fig. 2(c), owing to the band-gap renormalization concentrating on different states. In the setup shown in Fig. 3, the band-gap renormalization has the most affect on the A exciton states (pumping resonantly A excitons) and only few excitons are scattered to the B states, while in the setup in Fig. 2(c), hot carriers concentrate on states around the $\mathrm{B}$ excitons (pumping resonantly B excitons).

The differential reflection spectrum [Fig. 3(b)] also reveals another transition when the probing energy is around $2.32 \mathrm{eV}$. A typical $\Delta R$ curve is shown in Fig. 3(c). A positive differential reflection usually results from the band filling effect, implying that the transition is likely associated with the continuum state of the A exciton, i.e., the free carriers from the band edge transition. A portion of the pumped A excitons could be excited to higher excited states and eventually could be ionized to free carriers due to the exciton-exciton annihilation, phonon scattering, or local electric field originating from defects. The ionized electrons and holes occupy the quasiparticle electronic band edges. Note that the population of $\mathrm{C}$ excitons upon pumping around the A exciton states is negligible owing to the large energy and momentum separation [45]. The relaxation process, shown by the time-resolved reflectance spectrum in Fig. 3(c), is fitted by a biexponential decay function. The reflectance signal decays with a time constant of $130 \pm 13$ ps after a rapid decay characterized by a time constant of $8 \pm 1$ ps. The fast decay likely originates from the defect filling, as the ionized carriers rather than electrically neutral excitons experience strong long-range Coulomb interactions induced from local or even remote charge traps. Once the strong local electric field is subsidized, the slow component is prominent.
The probing-energy-dependent Kerr rotation spectrum at zero time delay shown in Fig. 3(a) reads a significant Kerr signal across that area. This also rules out the potential detection of high energy excitons ( $\mathrm{C}$ excitons) around the $\Gamma$ points in their Brillouin zone as the valley-dependent optical selection rules are not valid around the Brillouin zone center. So it is concluded that the transition around $2.32 \mathrm{eV}$ corresponds to the quasiparticle band edge. Compared to
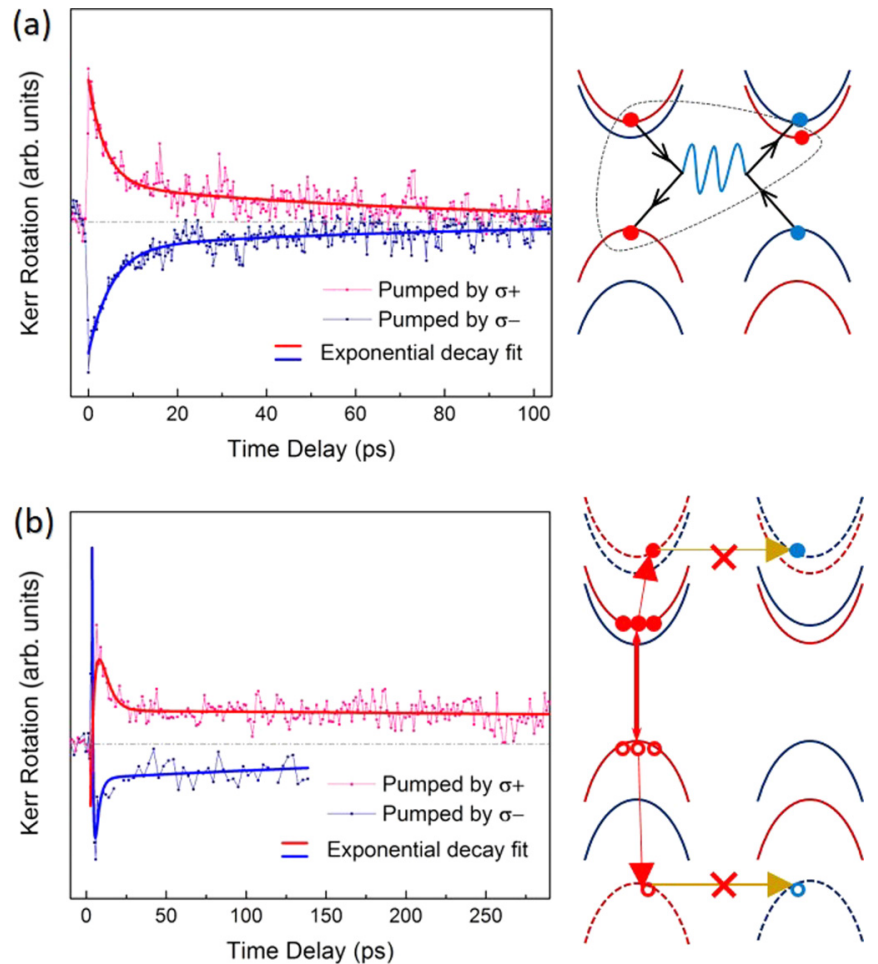

FIG. 4. (a) Time-resolved Kerr rotation of monolayer $\mathrm{WSe}_{2}$ pumped at $1.8 \mathrm{eV}$ and probed at $1.71 \mathrm{eV}$ (trion) at $70 \mathrm{~K}$. The illustration of negatively charged exciton valley relaxation is shown on the right-hand side. (b) Kerr rotation pumped at $1.737 \mathrm{eV}$ and probed at $2.318 \mathrm{eV}$ at $70 \mathrm{~K}$, and the sketch of the band structure with the dashed lines indicates the quasiparticle bands without the modification of excitonic effects. 
the PL result, the exciton binding energy is extracted to be $0.596 \mathrm{eV}$, close to the values reported by other methods $[55,56]$. The free interband transition has a relatively weak optical oscillator strength and is totally submerged with the broad $\mathrm{C}$ excitons in the absorption spectrum shown in Fig. 1(c), while in our pump-probe measurement, only the band edge (A excitons) is pumped and the high energy state $\mathrm{C}$ excitons are not populated, so the signal from the $\mathrm{C}$ excitons fades and only a free interband appears in the pump-probe spectrum. This is one advantage of the pump-probe technique in exciton binding energy measurements.

Figures 4(a) and 4(b) show the time-resolved Kerr rotation spectra of trions and free carriers under pumping A excitons. The trion valley polarization (probing at $1.71 \mathrm{eV}$ ) relaxes significantly slower than that of A excitons, qualitatively consistent with previous reports [26-28]. A two-section exponential fit gives time constants of $5 \pm 1$ and $80 \pm 14$ ps, respectively. The valley polarization of free carriers (probing at $2.318 \mathrm{eV}$ ) experiences a rapid decay near zero time delay, followed by an even slower decay, with time constants of $5 \pm 2$ ps and $2.4 \pm 1 \mathrm{~ns}$, respectively. The large error results from the relatively short delay line limited by the experimental conditions. The three orders of magnitude increase of the valley relaxation time at free carrier states originates from the suppressed valley relaxation channel via electron-hole exchange interactions. The increased spatial separation between free electrons and holes dramatically weakens the electron-hole exchange interactions which dominate the valley relaxation of excitons. Considering the electron-hole symmetry and the large spin splitting in the valence band, we attribute the Kerr rotation signal to the valley polarization of holes.

Note that both the rapid relaxation channel of the trions and the continuum states share a similar fast decay process after initial pumping, with a time constant similar to the A excitons. Thus, we infer that the valley polarization of high-density trions and free carriers decays rapidly via band edge exciton states. It is suggested that the trion's valley relaxation likely goes through a similar channel as excitons, the electron-hole exchange interactions as illustrated in Fig. 4(a). The prolonged valley relaxation time of trions likely results from the weaker Coulomb interaction compared to that in excitons, while this relaxation channel is invalid for free carriers.

In conclusion, monolayer $\mathrm{WSe}_{2}$ is examined by the timeresolved Kerr rotation technique. We have experimentally revealed the exciton binding energy to be $0.60 \mathrm{eV}$. The valley relaxation time constants of excitons, trions, and free carriers are derived to be approximately $2 \mathrm{ps}, 80 \mathrm{ps}$, and $2 \mathrm{~ns}$ at $70 \mathrm{~K}$, respectively. Our observations of valley relaxation in monolayer $\mathrm{WSe}_{2}$ provide insights into the valley dynamics in monolayer TMDs and valleytronics development.

The work is financially supported by Area of Excellency (AoE/P-04/08), GRF (17317316 and 17300415) of University Grants Committee of Hong Kong.
[1] A. Rycerz, J. Tworzydło, and C. W. J. Beenakker, Valley filter and valley valve in graphene, Nat. Phys. 3, 172 (2007).

[2] D. Xiao, W. Yao, and Q. Niu, Valley-Contrasting Physics in Graphene: Magnetic Moment and Topological Transport, Phys. Rev. Lett. 99, 236809 (2007).

[3] Y. P. Shkolnikov, E. P. De Poortere, E. Tutuc, and M. Shayegan, Valley Splitting of AlAs Two-Dimensional Electrons in a Perpendicular Magnetic Field, Phys. Rev. Lett. 89, 226805 (2002).

[4] D. Xiao, M.-C. Chang, and Q. Niu, Berry phase effects on electronic properties, Rev. Mod. Phys. 82, 1959 (2010).

[5] D. Xiao, G.-B. Liu, W. Feng, X. Xu, and W. Yao, Coupled Spin and Valley Physics in Monolayers of $\mathrm{MoS}_{2}$ and Other Group-VI Dichalcogenides, Phys. Rev. Lett. 108, 196802 (2012).

[6] H. Zeng, J. Dai, W. Yao, D. Xiao, and X. Cui, Valley polarization in $\mathrm{MoS}_{2}$ monolayers by optical pumping, Nat. Nanotechnol. 7, 490 (2012)

[7] T. Cao, G. Wang, W. Han, H. Ye, C. Zhu, J. Shi, Q. Niu, P. Tan, E. Wang, B. Liu, and J. Feng, Valley-selective circular dichroism of monolayer molybdenum disulphide, Nat. Commun. 3, 887 (2012).

[8] K. F. Mak, K. He, J. Shan, and T. F. Heinz, Control of valley polarization in monolayer $\mathrm{MoS}_{2}$ by optical helicity, Nat. Nanotechnol. 7, 494 (2012).

[9] G. Sallen, L. Bouet, X. Marie, G. Wang, C. R. Zhu, W. P. Han, Y. Lu, P. H. Tan, T. Amand, B. L. Liu, and B. Urbaszek, Robust optical emission polarization in $\mathrm{MoS}_{2}$ monolayers through selective valley excitation, Phys. Rev. B 86, 081301(R) (2012).
[10] H. Zeng, G.-B. Liu, J. Dai, Y. Yan, B. Zhu, R. He, L. Xie, S. Xu, X. Chen, W. Yao, and X. Cui, Optical signature of symmetry variations and spin-valley coupling in atomically thin tungsten dichalcogenides, Sci. Rep. 3, 1608 (2013).

[11] H. Yuan, M. S. Bahramy, K. Morimoto, S. Wu, K. Nomura, B.-J. Yang, H. Shimotani, R. Suzuki, M. Toh, C. Kloc et al., Zeeman-type spin splitting controlled by an electric field, Nat. Phys. 9, 563 (2013).

[12] Z. Gong, G.-B. Liu, H. Yu, D. Xiao, X. Cui, X. Xu, and W. Yao, Magnetoelectric effects and valley-controlled spin quantum gates in transition metal dichalcogenide bilayers, Nat. Commun. 4, 2053 (2013).

[13] A. Srivastava, M. Sidler, A. V. Allain, D. S. Lembke, A. Kis, and A. Imamoğlu, Valley Zeeman effect in elementary optical excitations of monolayer $\mathrm{WSe}_{2}$, Nat. Phys. 11, 141 (2015).

[14] D. MacNeill, C. Heikes, K. F. Mak, Z. Anderson, A. Kormányos, V. Zólyomi, J. Park, and D. C. Ralph, Breaking of Valley Degeneracy by Magnetic Field in Monolayer $\mathrm{MoSe}_{2}$, Phys. Rev. Lett. 114, 037401 (2015).

[15] G. Aivazian, Z. Gong, A. M. Jones, R.-L. Chu, J. Yan, D. G. Mandrus, C. Zhang, D. Cobden, W. Yao, and X. Xu, Magnetic control of valley pseudospin in monolayer $\mathrm{WSe}_{2}$, Nat. Phys. 11, 148 (2015).

[16] L. Xie and X. Cui, Manipulating spin-polarized photocurrents in 2D transition metal dichalcogenides, Proc. Natl. Acad. Sci. USA 113, 3746 (2016).

[17] Q. Wang, S. Ge, X. Li, J. Qiu, Y. Ji, J. Feng, and D. Sun, Valley carrier dynamics in monolayer molybdenum disulfide 
from helicity-resolved ultrafast pump-probe spectroscopy, ACS Nano 7, 11087 (2013).

[18] C. Mai, A. Barrette, Y. Yu, Y. G. Semenov, K. W. Kim, L. Cao, and K. Gundogdu, Many-body effects in valleytronics: Direct measurement of valley lifetimes in single-layer $\mathrm{MoS}_{2}$, Nano Lett. 14, 202 (2014).

[19] N. Kumar, J. He, D. He, Y. Wang, and H. Zhao, Valley and spin dynamics in $\mathrm{MoSe}_{2}$ two-dimensional crystals, Nanoscale 6, 12690 (2014)

[20] G. Plechinger, P. Nagler, C. Schüller, and T. Korn, Time-resolved Kerr rotation spectroscopy of valley dynamics in single-layer $\mathrm{MoS}_{2}$, arXiv:1404.7674.

[21] D. Lagarde, L. Bouet, X. Marie, C. R. Zhu, B. L. Liu, T. Amand, P. H. Tan, and B. Urbaszek, Carrier and Polarization Dynamics in Monolayer $\mathrm{MoS}_{2}$, Phys. Rev. Lett. 112, 047401 (2014).

[22] G. Wang, L. Bouet, D. Lagarde, M. Vidal, A. Balocchi, T. Amand, X. Marie, and B. Urbaszek, Valley dynamics probed through charged and neutral exciton emission in monolayer $\mathrm{WSe}_{2}$, Phys. Rev. B 90, 075413 (2014).

[23] C. R. Zhu, K. Zhang, M. Glazov, B. Urbaszek, T Amand, Z. W. Ji, B. L. Liu, and X. Marie, Exciton valley dynamics probed by Kerr rotation in $\mathrm{WSe}_{2}$ monolayers, Phys. Rev. B 90, 161302 (2014).

[24] G. Plechinger, P. Nagler, A. Arora, R. Schmidt, A. Chernikov, A. Granados Del Águila, P. C. M. Christianen, R. Bratschitsch, C. Schüller, and T. Korn, Trion fine structure and coupled spinvalley dynamics in monolayer tungsten disulfide, Nat. Commun. 7, 12715 (2016).

[25] A. Singh, K. Tran, M. Kolarczik, J. Seifert, Y. Wang, K. Hao, D. Pleskot, N. M. Gabor, S. Helmrich, N. Owschimikow, U. Woggon, and X. Li, Long-Lived Valley Polarization of Intravalley Trions in Monolayer WSe ${ }_{2}$, Phys. Rev. Lett. 117, 257402 (2016).

[26] L. Yang, N. A. Sinitsyn, W. Chen, J. Yuan, J. Zhang, J. Lou, and S. A. Crooker, Long-lived nanosecond spin relaxation and spin coherence of electrons in monolayer $\mathrm{MoS}_{2}$ and $\mathrm{WS}_{2}$, Nat. Phys. 11, 830 (2015).

[27] L. Yang, W. Chen, K. M. McCreary, B. T. Jonker, J. Lou, and S. A. Crooker, Spin coherence and dephasing of localized electrons in monolayer $\mathrm{MoS}_{2}$, Nano Lett. 15, 8250 (2015).

[28] W.-T. Hsu, Y.-L. Chen, C.-H. Chen, P.-S. Liu, T.-H. Hou, L.-J. Li, and W.-H. Chang, Optically initialized robust valley-polarized holes in monolayer $\mathrm{WSe}_{2}$, Nat. Commun. 6, 8963 (2015).

[29] X. Song, S. Xie, K. Kang, J. Park, and V. Sih, Long-lived hole spin/valley polarization probed by Kerr rotation in monolayer $\mathrm{WSe}_{2}$, Nano Lett. 16, 5010 (2016).

[30] E. J. Bushong, Y. (K.) Luo, K. M. McCreary, M. J. Newburger, S. Singh, B. T. Jonker, and R. K. Kawakami, Imaging spin dynamics in monolayer $\mathrm{WS}_{2}$ by time-resolved Kerr rotation microscopy, arXiv:1602.03568.

[31] D. Y. Qiu, F. H. da Jornada, and S. G. Louie, Optical Spectrum of $\mathrm{MoS}_{2}$ : Many-Body Effects and Diversity of Exciton States, Phys. Rev. Lett. 111, 216805 (2013).

[32] K. He, N. Kumar, L. Zhao, Z. Wang, K. F. Mak, H. Zhao, and J. Shan, Tightly Bound Excitons in Monolayer $\mathrm{WSe}_{2}$, Phys. Rev. Lett. 113, 026803 (2014).

[33] B. Zhu, X. Chen, and X. Cui, Exciton binding energy of monolayer $\mathrm{WS}_{2}$, Sci. Rep. 5, 9218 (2015).

[34] Z. Ye, T. Cao, K. O'Brien, H. Zhu, X. Yin, Y. Wang, S. G. Louie, and $\mathrm{X}$. Zhang, Probing excitonic dark states in single-layer tungsten disulphide, Nature (London) 513, 214 (2014).

[35] A. Chernikov, T. C. Berkelbach, H. M. Hill, A. Rigosi, Y. Li, O. B. Aslan, D. R. Reichman, M. S. Hybertsen, and T. F. Heinz, Exciton Binding Energy and Nonhydrogenic Rydberg Series in Monolayer $\mathrm{WS}_{2}$, Phys. Rev. Lett. 113, 076802 (2014).

[36] M. M. Ugeda, A. J Bradley, S.-F. Shi, F. H. da Jornada, Y. Zhang, D. Y. Qiu, W. Ruan, S.-K. Mo, Z. Hussain, Z.-X. Shen et al., Giant bandgap renormalization and excitonic effects in a monolayer transition metal dichalcogenide semiconductor, Nat. Mater. 13, 1091 (2014).

[37] C. Zhang, A. Johnson, C.-L. Hsu, L.-J. Li, and C.-K. Shih, Direct imaging of band profile in single layer $\mathrm{MoS}_{2}$ on graphite: Quasiparticle energy gap, metallic edge states, and edge band bending, Nano Lett. 14, 2443 (2014).

[38] A. M. Jones, H. Yu, N. J. Ghimire, S. Wu, G. Aivazian, J. S. Ross, B. Zhao, J. Yan, D. G. Mandrus, D. Xiao et al., Optical generation of excitonic valley coherence in monolayer $\mathrm{WSe}_{2}$, Nat. Nanotechnol. 8, 634 (2013).

[39] T. Yu and M. W. Wu, Valley depolarization due to intervalley and intravalley electron-hole exchange interactions in monolayer $\mathrm{MoS}_{2}$, Phys. Rev. B 89, 205303 (2014).

[40] M. M. Glazov, T. Amand, X. Marie, D. Lagarde, L. Bouet, and B. Urbaszek, Exciton fine structure and spin decoherence in monolayers of transition metal dichalcogenides, Phys. Rev. B 89, 201302(R) (2014).

[41] T. Yan, X. Qiao, P. Tan, and X. Zhang, Valley depolarization in monolayer $\mathrm{WSe}_{2}$, Sci. Rep. 5, 15625 (2015).

[42] T. Yan, J. Ye, X. Qiao, P. Tan, and X. Zhang, Exciton valley dynamics in monolayer $\mathrm{WSe}_{2}$ probed by the two-color ultrafast Kerr rotation, Phys. Chem. Chem. Phys. 19, 3176 (2017).

[43] W. Zhao, Z. Ghorannevis, L. Chu, M. Toh, C. Kloc, P.-H. Tan, and G. Eda, Evolution of electronic structure in atomically thin sheets of $\mathrm{WS}_{2}$ and $\mathrm{WSe}_{2}$, ACS Nano 7, 791 (2012).

[44] A. Carvalho, R. M. Ribeiro, and A. H. Castro Neto, Band nesting and the optical response of two-dimensional semiconducting transition metal dichalcogenides, Phys. Rev. B 88, 115205 (2013).

[45] D. Kozawa, R. Kumar, A. Carvalho, K. K. Amara, W. Zhao, S. Wang, M. Toh, R. M. Ribeiro, A. H. Castro Neto, K. Matsuda et al., Photocarrier relaxation pathway in two-dimensional semiconducting transition metal dichalcogenides, Nat. Commun. 5, 4543 (2014).

[46] I. Kylänpää and H.-P. Komsa, Binding energies of exciton complexes in transition metal dichalcogenide monolayers and effect of dielectric environment, Phys. Rev. B 92, 205418 (2015).

[47] See Supplemental Material at http://link.aps.org/supplemental/ 10.1103/PhysRevB.95.241406 for details of laser pulse broadening analysis, Kerr rotation measurement, steady-state spectra comparison on the $\mathrm{SiO}_{2}$ and mica substrates, fitting method, pump energy dependent Kerr rotation, and pump fluence dependent exciton and valley dynamics.

[48] T. Smoleński, M. Goryca, M. Koperski, C. Faugeras, T. Kazimierczuk, A. Bogucki, K. Nogajewski, P. Kossacki, and M. Potemski, Tuning Valley Polarization in a $\mathrm{WSe}_{2}$ Monolayer with a Tiny Magnetic Field, Phys. Rev. X 6, 021024 (2016).

[49] M. Baranowski, A. A. Surrente, D. K. Maude, M. Ballottin, A. Mitioglu, P. C. M. Christianen, Y. C. Kung, D. Dumcenco, A. Kis, and P. Plochocka, Dark excitons and the elusive valley 
polarization in transition metal dichalcogenides, 2D Mater. 4, 025016 (2017).

[50] X.-X. Zhang, T. Cao, Z. Lu, Y.-C. Lin, F. Zhang, Y. Wang, Z. Li, J. C. Hone, J. A. Robinson, D. Smirnov et al., Magnetic brightening and control of dark excitons in monolayer $\mathrm{WSe}_{2}$, arXiv:1612.03558.

[51] D. Sun, Y. Rao, G. A. Reider, G. Chen, Y. You, L. Brézin, A. R. Harutyunyan, and T. F. Heinz, Observation of rapid exciton-exciton annihilation in monolayer molybdenum disulfide, Nano Lett. 14, 5625 (2014).

[52] D. A. Kleinman and R. C. Miller, Band-gap renormalization in semiconductor quantum wells containing carriers, Phys. Rev. B 32, 2266 (1985).

[53] G. Tränkle, H. Leier, A. Forchel, H. Haug, C. Ell, and G. Weimann, Dimensionality Dependence of the Band-Gap
Renormalization in Two- and Three-Dimensional Electron-Hole Plasmas in GaAs, Phys. Rev. Lett. 58, 419 (1987).

[54] M. Manca, M. M. Glazov, C. Robert, F. Cadiz, T. Taniguchi, K. Watanabe, E. Courtade, T. Amand, P. Renucci, X. Marie et al., Enabling valley selective exciton scattering in monolayer $\mathrm{WSe}_{2}$ through upconversion, Nat. Commun. 8, 14927 (2017).

[55] G. Wang, X. Marie, I. Gerber, T. Amand, D. Lagarde, L. Bouet, M. Vidal, A. Balocchi, and B. Urbaszek, Giant Enhancement of the Optical Second-Harmonic Emission of $\mathrm{WSe}_{2}$ Monolayers by Laser Excitation at Exciton Resonances, Phys. Rev. Lett. 114, 097403 (2015).

[56] A. T. Hanbicki, M Currie, G. Kioseoglou, A. L. Friedman, and B. T. Jonker, Measurement of high exciton binding energy in the monolayer transition-metal dichalcogenides $\mathrm{WS}_{2}$ and $\mathrm{WSe}_{2}$, Solid State Commun. 203, 16 (2015). 